\title{
$\$$ Research Square

\section{Background factors affecting visual acuity at initial visit in eyes with central retinal vein occlusion: multicenter study in Japan}

Mineo Kondo ( $\square$ mineo@clin.medic.mie-u.ac.jp )

Mie University Graduate School of Medicine

Hidetaka Noma

Tokyo Medical University Hachioji Medical Center

Masahiko Shimura

Tokyo Medical University Hachioji Medical Center

Masahiko Sugimoto

Mie University Graduate School of Medicine

Yoshitsugu Matsui

Mie University Graduate School of Medicine

Yoshitsugu Saishin

Shiga University of Medical Science

Masahito Ohji

Shiga University of Medical Science

Hiroto Ishikawa

Hyogo College of Medicine

Fumi Gomi

Hyogo College of Medicine

Kensaku Iwata

Kurume University School of Medicine

Shigeo Yoshida

Kurume University School of Medicine

Sentaro Kusuhara

Kobe University Graduate School of Medicine

Hiromasa Hirai

Nara Medical University Graduate School of Medicine

Nahoko Ogata

Nara Medical University Graduate School of Medicine

Takao Hirano

Shinshu University School of Medicine

Toshinori Murata 
Shinshu University School of Medicine

Kotaro Tsuboi

Aichi Medical University

Motohiro Kamei

Aichi Medical University

\section{Takamasa Kinoshita}

Sapporo City General Hospital

\section{Soichiro Kuwayama}

Nagoya City University Graduate School of Medical Sciences

\section{Yoshio Hirano}

Nagoya City University Graduate School of Medical Sciences

\section{Manami Ohta}

Yamaguchi University Graduate School of Medicine

\section{Kazuhiro Kimura}

Yamaguchi University Graduate School of Medicine

\section{Kei Takayama}

National Defense Medical College

\section{Masaru Takeuchi}

National Defense Medical College

\section{Yoshihiro Takamura}

University of Fukui

\section{Fumiki Okamoto}

University of Tsukuba

\section{Yoshinori Mitamura}

Tokushima University Graduate School

\section{Hiroto Terasaki}

Kagoshima University Graduate School of Medical and Dental Sciences

\section{Taiji Sakamoto}

Kagoshima University Graduate School of Medical and Dental Sciences

\section{Research Article}

Keywords: visual acuity, central retinal vein occlusion, multicenter study, baseline characteristics

Posted Date: August 18th, 2021

DOI: https://doi.org/10.21203/rs.3.rs-815373/v1

License: (c) (P) This work is licensed under a Creative Commons Attribution 4.0 International License. Read Full License 
Version of Record: A version of this preprint was published at Journal of Clinical Medicine on November 29th, 2021. See the published version at https://doi.org/10.3390/jcm10235619. 


\section{Abstract}

This retrospective multicenter study was designed to determine the baseline characteristics of patients in Japan with central retinal vein occlusion (CRVO) that were significantly associated with best-corrected visual acuity (BCVA) at the initial examination. The patients' baseline factors that were significantly associated with the BCVA at the initial examination were determined by univariate, multivariate, and logistic regression analyses. Data from 517 eyes of 517 patients (mean age, $69.9 \pm 12.2$ years; men, $57.3 \%)$ were analyzed. Univariate analyses showed that an older age $(r=0.194, P<0.001)$ and the right eye $(r=-0.103, P<0.019)$ were significantly associated with poorer BCVA at the initial visit. Multivariate analyses showed that an older age $(\beta=0.191, P<0.001)$ and the right eye $(\beta=-0.089, P=0.041)$ were also significantly associated with poorer BCVA at the initial visit. In addition, logistic regression analyses showed that these two factors were also significantly related to the BCVAs poorer than 1.0 logMAR units ( 0.1 decimal BCVA) at the initial visit. Results indicate that an older age, a known strong factor, and the right eye were significantly associated with poorer BCVA at the initial visit. These results suggest that functional and/or anatomical differences between the right and left eyes may be involved in these results.

\section{Main Text}

A central retinal vein occlusion (CRVO) is a retinal vascular disorder that can result in severe vision reduction. The exact pathogenesis of CRVO has not been definitively determined but several factors including compression by a sclerotic central retinal artery, degenerative changes of the vessel wall, hemodynamic disturbances, blood hypercoagulability, and partial thrombosis may be involved. ${ }^{1-4}$ The global prevalence of CRVO is estimated at about $0.08-0.13 \%$ with higher incidence in older individuals. ${ }^{5-7}$ The common causes of vision reduction in CRVO are macular edema, vitreous hemorrhage due to retinal neovascularization, and rubeotic glaucoma due to iris/angle neovascularization.

At present, several different types of treatments have been used for eyes with CRVO including laser photocoagulation, ${ }^{8}$ intravitreal injection of corticosteroids including triamcinolone acetonide, ${ }^{9}$ intravitreal implant of dexamethasone, ${ }^{10}$ and intravitreal injections of anti-vascular endothelial growth factor (antiVEGF) agents ${ }^{11,12}$. However, the visual prognosis of CRVO is still not any better than that for branch retinal vein occlusion (BRVO). ${ }^{13-15}$

It is known that the baseline best-corrected visual acuity (BCVA) is the strongest predictor for the final BCVA in eyes with CRVO. ${ }^{16-20}$ However, a PubMed search did not extract any study examining the background factors of the patients that were significantly associated with poorer BCVAs at the initial visit to the hospital.

Thus, the purpose of this study was to determine the baseline characteristics of patients with a CRVO that were significantly associated with the baseline BCVA. To accomplish this, we conducted a multicenter collaborative study of 517 eyes of 517 patients to determine what background factors were 
significantly associated with poorer BCVA at the first examination in eyes with a CRVO. We shall show that in addition to age, a well-known strong factor, the affected eye (right or left eye) was significantly associated with the visual acuity at the initial visit to the hospital.

\section{Results}

Data of 588 eyes with a CRVO were collected from 17 ophthalmological institutions throughout Japan. Seventy-one eyes $(12 \%)$ were excluded due to inadequate inclusion criteria or missing data, and in the end, 517 eyes were used for the analyses.

\section{Demographic information}

Demographic information of the 517 eyes of 517 patients with a CRVO is shown in Table 1 . The mean \pm SD age of the patients was $69.9 \pm 12.2$ years with a range of 22 to 94 years. Fifty-eight percent of the patients were $\geq 70$ years (Fig. 1A). The mean age was significantly higher in women (71.9 \pm 12.1 years) than men $(68.4 \pm 12.1$ years, $P=0.001$, Fig. 1B). This difference was thought to be due to the longer life expectancy of women in Japan. Only 1.9\% (10 eyes) of all CRVO patients were under 40-years-of-age.

Of the 517 patients with CRVO, 296 were men $(57.3 \%)$ and 221 were women $(42.7 \%)$. The difference in probabilities of the presence of a CRVO between men and women was statistically significant $(P=0.001$, binominal test). Systemic hypertension was present in 334 patients $(64.6 \%)$, and 87 patients $(16.8 \%)$ had diabetes mellitus. The mean interval between the onset of symptoms to the initial visit to hospital was $6.4 \pm 6.9$ weeks (range, $0-51$ weeks). We also noted that 467 of 517 patients $(90.3 \%$ ) visited a hospital within 12 weeks of the onset of the symptoms.

The mean \pm SD of the BCVA was $0.72 \pm 0.55 \log M A R$ units $(0.19$ decimal BCVA) with a range of -0.18 to $2.30 \log M A R$ units. The number of eyes with a BCVA worse than $0.3 \log M A R$ units $(0.50$ decimal BCVA) was $355(88.7 \%)$, and the number of eyes with a BCVA worse than $1.0 \log M A R$ units ( 0.1 decimal BCVA) was $113(21.9 \%)$. The mean \pm SD central macular thickness (CMT) at the initial visit was $632 \pm 237 \mu \mathrm{m}$ with a range of 62 to $1456 \mu \mathrm{m}$. A CMT of $62 \mu \mathrm{m}$ was found in one patient with ischemic CRVO who visited a hospital 11 months after the onset of the symptoms, and the macula was severely atrophic.

At the initial visit, 122 eyes (23.6\%) were classified as the ischemic-type, 377 eyes (72.9\%) were the nonischemic-type, and 18 eyes (3.5\%) were unclassifiable.

\section{Background factors affecting visual acuity at initial visit}

To determine the patients' background factors that affected the BCVA at the initial visit to the hospital, we performed univariate and multivariate linear regression analyses (Table 2). Univariate linear regression analyses showed that the age $(r=0.194, P<0.001)$ and the affected eye (right or left, $r=-0.103, P=$ $0.019)$ were significantly associated with the BCVA at the initial visit to the hospital (Table 2, left panel). Multivariate linear regression analyses also identified the same two independent factors; the age $(\beta=$ 
$0.191, P<0.001)$ and the affected eye $(\beta=-0.089, P=0.041)$ as independent factors which affected the BCVA at the initial visit to the hospital (Table 2, right panel).

We also sought to identify the background factors that were significantly related to the BCVA worse than $1.0 \log$ MAR units ( 0.1 decimal BCVA) at the initial visit to the hospital using logistic regression analyses (Suppl. Table 1). The same six dependent values were used for the independent variables. Results showed that the same two factors, an older age $(\beta=0.243, P=0.016)$ and the right eye $(\beta=-0.195, P=$ 0.038 ) were found to be significantly associated with a BCVA worse than $1.0 \log$ MAR units at the initial visit.

\section{Relationship between age and BCVA at initial visit to hospital}

The BCVAs (logMAR units) at the initial visit to the hospital are plotted against the age in Figure 2A. A linear regression fit to the data indicated that a 10-year increase of age was accompanied by an approximately -0.0876 logMAR unit change of the BCVA at the initial visit to the hospital (red line, Fig. 2A).

A plot of the BCVA at the initial visit to hospital for each age group showed that the mean BCVA was 0.50 logMAR units ( 0.32 decimal BCVA) for the eyes $<50$ years. The mean BCVA became gradually worse with age and reached to $0.83 \log$ MAR units ( 0.15 decimal BCVA) for eyes of patients $\geq 80$ years (Fig. 2B).

\section{Comparisons of visual acuity and central macular thickness between right and left eyes}

Because both linear and logistic regression analyses demonstrated that the affected eye (right or left) was significantly associated with the BCVA at the initial visit to the hospital, we next plotted the BCVA of the right and left eyes in all 517 CRVO patients, (Fig. 3A, left). The mean \pm SD BCVA (logMAR units) at the initial visit to the hospital was significantly better in the left eye $(0.66 \pm 0.53$ logMAR units) than in the right eye $(0.78 \pm 0.56$, logMAR units; $P=0.019)$. We also noted that the percentage of eyes with BCVA worse than 1.0 logMAR ( 0.1 decimal BCVA) was $30.0 \%$ (72/240 eyes) in the right eye, whereas it was $22.7 \%$ in the left eye $(63 / 277$ eyes).

We also compared the CMT of the right and left eyes at the initial visit and found that the mean CMT was significantly thicker in the right eye $(655 \pm 253 \mu \mathrm{m})$ than in the left eye $(613 \pm 218 \mu \mathrm{m}, P=0.011$; Fig. 3B). There was also a significant correlation between the BCVA and CMT at the initial examination (Supp. Fig. 1).

The percentage of ischemic CRVO were also compared between the right and left eyes. The percentage of ischemic CRVO tended to be slightly higher in the right eye $(25.8 \%, 62 / 240$ eyes) than in the left eye $(21.7 \%, 60 / 277$ eyes), but this difference was not statistically significant $(P=0.319$, chi-square test).

\section{Discussion}


In this multicenter study, we examined the background characteristics of patients with CRVO in Japan and sought to determine the patients' background factors which were significantly associated with the BCVA at the initial examination. The background characteristics of the patients with CRVO were similar to recent real-world data of CRVO: the average age of the patients was about 70 years and patients had a high prevalence of hypertension. ${ }^{13,15,19,21,22}$

In our CRVO cohort, there were significantly more men (57.3\%) than women $(42.7 \%, P<0.001$, Table 1$)$. Another recent study of CRVO in Japan also showed that the prevalence was higher in men (64.7\%) than women (35.3\%), ${ }^{19}$ whereas the global epidemiological studies have shown that the prevalence of CRVO did not differ significantly between sexes. ${ }^{5,7}$ Recent real-world reports of CRVO in USA, ${ }^{15} \mathrm{UK}^{21}$ and Germany ${ }^{13}$ also reported almost equal prevalence of CRVO between men and women. We presume that the higher prevalence of CRVO in men may be related to higher prevalence of hypertension and smoking rate of men in Japan. ${ }^{23,24}$ Analyses of data from National Surveys of Japan have shown that there was a clear trend for a decrease in the prevalence of hypertension in women, but not in men over the past 30 years in Japan. ${ }^{25}$

We also found that an older age was associated with poorer BCVA at the initial visit to the hospital. This was not too surprising because there have been many past studies reporting that an older age was associated with worse final visual outcomes in eyes with CRVO. ${ }^{16,18-20}$ We presume that the age-related sclerotic changes in the arteries and the degenerative changes of the vessel wall may be associated with this result. On the other hand, we cannot rule out the possibility that the age-related mild cataract might have affected our results because we excluded CRVO patients with only severe cataracts ( $\geq$ grade 3 ) in this study.

The most interesting and unexpected findings in this study was that the right to left eye difference affected the baseline BCVA at initial examination in eyes with CRVO. Both univariate and multivariate linear regression analyses demonstrated that the right eye was significantly associated with poorer BCVA at the initial visit to the hospital in eyes with CRVO (Table 2 \& Fig. 3A). We also found that the right eye was significantly related to the initial BCVA poorer than 1.0 logMAR (0.1 decimal BCVA; Supp. Table 1). This difference in the BCVA between the right and left eyes appeared to be related to the degree of macular edema because the CMT was also significantly higher in the right eye than in the left eye (Fig. 3B), and there was a significant correlation between the visual acuity and the CMT (Supp. Fig. 1).

Our PubMed search showed that our findings are the first report of a significant difference in the BCVA between the right and the left eyes in eyes with CRVO. The exact reason for the difference in the initial BCVA between the right and left eyes in CRVO was not determined. We initially suspected that the right eyes had a higher frequency of the ischemic-type of CRVO. Although, the ratios of ischemic CRVO tended to be slightly higher in the right eye (25.8\%) than in the left eye $(21.7 \%)$, this difference was not statistically significant $(P=0.319)$. 
The difference in the severity of CRVO between the right and left eyes may be due to some functional and/or anatomical differences between the right and left eyes. For example, it is known that the blood pressure measured on the right arm is slightly, but significantly, higher than that on the left arm. ${ }^{26,27}$ Some investigators have suggested that the blood pressure might be higher in the right brachial artery compared to the left because the brachiocephalic artery is located nearer the source of pressure and is more in the direction of flow of blood from the ascending aorta. ${ }^{28}$ These slight differences in blood pressure between the right and left circulations might cause different degrees of compressions by the central retinal artery.

Another possible reason for the difference in the initial visual acuity of eyes with a CRVO between the right and left eye may be the anatomical difference in the arteries of the right and left eyes. For example, there is a past study reporting that the diameter of the carotid artery tended to be larger in the right than the left carotid artery $(P=0.051) .{ }^{29}$ In addition, it has been reported that the diameter of the ophthalmic artery and central retinal artery tended to be larger on the right than on the left sides, although these differences were not statistically significant. ${ }^{30}$ If the diameter of the arteries supplying blood to the right eye were larger in the right eye than in the left eye, the degree of compression of the central retinal vein by the central retinal artery might be higher. This then might have caused the CRVO in the right eyes to be more severe.

There are several limitations in this study. First limitation is the retrospective nature of this study. In addition, we analyzed the factors affecting the baseline BCVA using only six background factors of the patients because we examined the medical records retrospectively. If other factors such as smoking, hyperlipidemia or arterial thromboembolic diseases could have been included, a more detailed analysis would have been possible.

The second limitation is that all subjects were of the same ethnicity, i.e., Japanese. The results obtained in this study may be specific for the Japanese. Further studies are needed to verify whether the left-right difference can be a significant factor affecting the initial visual acuity of CRVO in patients of other ethnicities.

A third limitation was that we did not analyze the factors which significantly influenced final visual outcomes in CRVO. We did not do this because many other factors including the initial BCVA and kinds of treatments for CRVO would need to be considered to determine which were related to the final visual outcome. However, we did find that the difference in the BCVA between the right and the left eyes was still nearly significant even at 12 months after the treatments (right, $0.62 \pm 0.61$; left $0.50 \pm 0.54, P=0.055$ ). We plan to present these results in the next paper in more detail.

In summary, we have presented the background characteristics of patients with CRVO in Japan and studied the patients' background factors which were significantly associated with the BCVA at the initial examination. We found that in addition to age, which is factor known to affect the BCVA in eyes with CRVO, the right and left eye differences can be another possible factors affecting the visual acuity at the 
initial visit. Although the mechanism for this difference still remains undetermined, the results suggest a new background factor which can influence the initial and final visual acuities in eyes with CRVO.

\section{Methods}

\section{Study design and approval}

This was a multicenter retrospective study using medical records of 30 retina specialists at 17 ophthalmological institutions in Japan. The study protocol was approved by the Ethics Committees of all participating centers with the main Ethics Committee at the Mie University Hospital (\#H2018-034). A written informed consent was not obtained from the subjects because of the retrospective nature of this study. Instead, a home page was created with information on the purpose of this study for the subjects to read. We emphasized that any subject could opt out of the study at any time by telephone, fax, or e-mail. The study was also registered on the International Clinical Trial Registry Platform (UMIN Clinical Trials Registry, R000037330, http://www.umin.ac.jp/ctr/index-j.htm). The data acquired for the analysis were anonymized before they were examined. The procedures used conformed to the tenets of the Declaration of Helsinki of the World Medical Association.

\section{Subjects}

Treatment-naïve CRVO patients who visited the hospital between January 2013 and December 2017 and were followed for more than 12 months were enrolled in this study. Only CRVO patients whose age was $\geq 20$ years, and whose interval between the onset of symptoms to the initial visit to the hospital was $\leq 12$ month were included. Eyes with hemi-CRVO were excluded. CRVO eyes with glaucoma or advanced cataract ( $\geq$ Grade 3$)^{31}$ were also excluded. If patients had CRVO in both eyes and visited the hospital between January 2013 and December 2017, only the eye with an earlier onset was studied.

The diagnosis of CRVO was made by each retinal specialist based on the presence of retinal edema, optic disc hyperemia or edema, scattered superficial and deep retinal hemorrhages, and venous dilation using fundus photographs and optical coherence tomographic images according to earlier studies. ${ }^{5}$

The age, sex, affected eye (right or left), presence of systemic hypertension and diabetes mellitus, and the interval between symptoms onset to initial visit to the hospital were collected from the medical records. The BCVA and central macular thickness (CMT) at the initial visit were also collected.

\section{Visual acuity and central macular thickness}

The BCVA was measured with a standard Japanese decimal visual acuity chart at 5 meters. The visual acuities of "counting fingers" and "hand motion" were quantified to decimal values as 0.014 and 0.004 , respectively, based on the past study. ${ }^{32}$ The decimal values were converted to the logarithm of the minimal angle of resolution (logMAR) units for the statistical analyses. 
The CMT was measured by spectral-domain optical coherence tomography (OCT) at the initial visit using Spectralis (Heiderbelg Engineering, Heidelberg, Germany), Cirrus (Carl Zeiss Meditec, Inc, Dubrin, CA), Triton (Topcon, Tokyo, Japan), or RS-3000 (Nidek, Gamagori, Japan). The average thickness within a 1$\mathrm{mm}$ diameter of the central macular area was used for the analysis. If the average thickness within the 1$\mathrm{mm}$ diameter was not measured, the average foveal thickness of the vertical and horizontal scans was calculated and used as the value of the CMT.

\section{Classification of ischemic status}

All CRVO eyes were classified as the ischemic-type or nonischemic-type based on the findings of the fluorescein angiography (FA) performed at the initial visit. The classical definition of the CVO Study ${ }^{8}$ was used: the CRVO was classified as the ischemic-type if the eye had at least a 10-disc area of retinal capillary nonperfusion within the area of a standard photographic field. If FA was not performed, the eye was classified as ischemic-type when at least two of the following five findings were present; (1) massive retinal hemorrhage associated with prominent tortuosity of the retinal vessel, (2) decimal BCVA $<0.1,(3)$ loss of the 1-2e isopter in Goldmann visual field, (4) apparent relative afferent pupillary defect, or (5) ERG b-wave amplitude reduced to $\leq 60 \%$ of that of the normal fellow eye. ${ }^{33-35}$ This classification was done at the individual centers.

\section{Statistical analyses}

The probabilities of the appearance of a CRVO between men and women or between right and left eyes were compared by binomial tests. Univariate and multivariate linear regression analyses were used to determine the background factors which affected the BCVA at the initial visit to the hospital. The BCVAs at the initial visit to the hospital were used as the dependent variables. The independent variables were the age, sex, affected eye (right or left), presence of systemic hypertension, presence of diabetes mellitus, and interval from symptom onset to initial visit to the hospital. The coefficients of correlation $(r)$ and $P$ values were calculated for the univariate linear regression analysis, and standardized partial regression coefficient $(\beta)$ and $P$-values were calculated for the multivariate linear regression analyses for the six independent variables. To determine the background factors which were associated with BCVA poorer than $1.0 \log M A R$ units ( 0.1 decimal BCVA) at the initial visit to the hospital, the logistic regression analysis using stepwise forward selection method was used. The same six independent valuables were used.

After confirming that the data were approximately normally distributed, the significance of the differences in the BCVA (logMAR unit) or CMT between the right and left eye were compared with unpaired $t$-tests. The results were considered statistically significant when $P$ was less than 0.05 . Analyses were performed with SPSS software (IBM SPSS Statistics 25; IBM Corp., Armonk, NY, USA).

\section{Declarations}


Acknowledgements: The authors thank Duco I. Hamasaki of the University of Miami for critical discussion and final manuscript revisions. They also thank Dr. Norio Sugimoto for medical statistical analyses.

Author contributions: Conception and design: M.K. Analysis and interpretation: M.K., H.N., M.S., M.S., Y.M., Y.S., M.O., H.I., F.G., K.I., S.Y., S.K., H.H., N.O., T.H., T.M., K.T., M.K., T.K., S.K., Y.H., M.O., K.K., K.T., M.T., Y.T., F.O., Y.M., H.T., and T.S. Data collection: M.K., H.N., M.S., M.S., Y.M., Y.S., M.O., H.I., F.G., K.I., S.Y., S.K., H.H., N.O., T.H., T.M., K.T., M.K., T.K., S.K., Y.H., M.O., K.K., K.T., M.T., Y.T., F.O., Y.M., H.T., and T.S. Obtained funding: M.K., H.N., M.S., M.S., Y.M., Y.S., M.O., H.I., F.G., K.I., S.Y., S.K., H.H., N.O., T.H., T.M., K.T., M.K., T.K., S.K., Y.H., M.O., K.K., K.T., M.T., Y.T., F.O., Y.M., H.T., and T.S. Overall responsibility: M.K., H.N., M.S., M.S., Y.M., Y.S., M.O., H.I., F.G., K.I., S.Y., S.K., H.H., N.O., T.H., T.M., K.T., M.K., T.K., S.K., Y.H., M.O., K.K., K.T., M.T., Y.T., F.O., Y.M., H.T., and T.S.

Competing interests: The authors declare no competing interests.

Financial supports: None

Data availability: All data generated or analyzed during this study are included in this published article and its Supplementary Information files.

\section{References}

1. Hayreh, S. S. So-called "central retinal vein occlusion": I. Pathogenesis, terminology, clinical features., 172, 1-13 (1976).

2. Green, W. R., Hutchins, C. C. C., Terry, J. M. \& G. M. \& Central retinal vein occlusion: A prospective histopathologic study of 29 eyes in 28 cases. Trans. Am. Ophthalmol. Soc, 79, 371-422 (1981).

3. Prisco, D. \& Marcucci, R. Retinal vein thrombosis: Risk factors, pathogenesis and therapeutic approach. Pathophysiol. Haemost. Thromb, 32, 308-311 (2002).

4. Rehak, M. \& Wiedemann, P. Retinal vein thrombosis: Pathogenesis and management. J. Thromb. Haemost, 8, 1886-1894 (2010).

5. Rogers, S. et al. International Eye Disease Consortium. The prevalence of retinal vein occlusion: Pooled data from population studies from the United States, Europe, Asia, and Australia., 117, 313319 (2010).

6. Ponto, K. A. et al. Prevalence and risk factors of retinal vein occlusion: The Gutenberg Health Study. J. Thromb. Haemost, 13, 1254-1263 (2015).

7. Song, P., Xu, Y., Zha, M., Zhang, Y. \& Rudan, I. Global epidemiology of retinal vein occlusion: A systematic review and meta-analysis of prevalence, incidence, and risk factors. J. Glob. Health, 9, 010427 (2019).

8. The Central Vein Occlusion Study Group N Report. A randomized clinical trial of early panretinal photocoagulation for ischemic central vein occlusion., 102, 1434-1444 (1955). 
9. Ip, M. S. et al. A randomized trial comparing the efficacy and safety of intravitreal triamcinolone with observation to treat vision loss associated with macular edema secondary to central retinal vein occlusion: The Standard Care vs Corticosteroid for Retinal Vein Occlusion (SCORE) study report 5. Arch. Ophthalmol, 127, 1101-1114 (2009).

10. Haller, J. A. et al. Dexamethasone intravitreal implant in patients with macular edema related to branch or central retinal vein occlusion twelve-month study results., 118, 2453-2460 (2011).

11. Campochiaro, P. A. et al. Sustained benefits from ranibizumab for macular edema following central retinal vein occlusion: Twelve-month outcomes of a phase III study., 118, 2041-2049 (2011).

12. Heier, J. S. et al. Intravitreal aflibercept injection for macular edema due to central retinal vein occlusion: Two-year results from the COPERNICUS study., 121, 1414-1420 (2014).

13. Callizo, J. et al. Real-world data: Ranibizumab treatment for retinal vein occlusion in the OCEAN Study. Clin. Ophthalmol, 13, 2167-2179 (2019).

14. Costa, J. V. et al. Macular edema secondary to retinal vein occlusion in a real-life setting: A multicenter, nationwide, 3-year follow-up study. Graefes. Arch. Clin. Exp. Ophthalmol, 259, 343-350 (2021).

15. Ciulla, T., Pollack, J. S. \& Williams, D. F. Visual acuity outcomes and anti-VEGF therapy intensity in macular oedema due to retinal vein occlusion: A real-world analysis of 15613 patient eyes. Br. J. Ophthalmol, 104, https://doi.org/https://doi:10.1136/bjophthalmol-2020-317337 (2021).

16. Glacet-Bernard, A. et al. Prognostic factors for retinal vein occlusion: Prospective study of 175 cases., 103, 551-560 (1996).

17. The Central Vein Occlusion Study Group. Natural history and clinical management of central retinal vein occlusion. Arch. Ophthalmol, 115, 486-491 (1997).

18. Hayreh, S. S., Podhajsky, P. A. \& Zimmerman, M. B. Natural history of visual outcome in central retinal vein occlusion., 118, 119-133 (2011).

19. Nagasato, D. et al. Factors associated with extremely poor visual outcomes in patients with central retinal vein occlusion. Sci. Rep, 10, 19667 (2020).

20. Sen, P. et al. Predictors of visual acuity outcomes after anti-vascular endothelial growth factor treatment for macular edema secondary to central retinal vein occlusion. Ophthalmol. Retina, 5, https://doi https://doi.org/10.1016/j.oret.2021.02.008 (2021).

21. Eleftheriadou, M., Nicholson, L. \& D'Alonzo, G. \& Addison. P. K. F. Real-life evidence for using a treatand-extend injection regime for patients with central retinal vein occlusion. Ophthalmol. Ther, 8, 289296 (2019).

22. Hogg, H. D. J. et al. Real-world visual and neovascularisation outcomes from anti-VEGF in central retinal vein occlusion. Ophthalmic Epidemiol, 28, 70-76 (2021).

23. Tsugane, S. Alcohol, smoking, and obesity epidemiology in Japan. J. Gastroenterol. Hepatol, 27 (Suppl 2), 121-126 (2012). 
24. Otani, K., Haruyama, R. \& Gilmour, S. Prevalence and correlates of hypertension among Japanese adults, 1975 to 2010. Int. J. Environ. Res. Public Health, 15, 1645 (2018).

25. Miura, K., Nagai, M. \& Ohkubo, T. Epidemiology of hypertension in Japan: Where are we now? Circ. J, 77, 2226-2231 (2013).

26. Cassidy, P. \& Jones, K. A study of inter-arm blood pressure differences in primary care. J. Hum. Hypertens, 15, 519-522 (2001).

27. Lane, D. et al. Inter-arm differences in blood pressure: when are they clinically significant? J. Hypertens, 20, 1089-1095 (2002).

28. Southby, R. Some clinical observations on blood pressure and their practical application, with special reference to variation of blood pressure readings in the two arms. Med. J. Aust, 2, 569-580 (1935).

29. Bos, M. J., van Loon, R. F., Heywood, L., Morse, M. P. \& van Zundert, A. A. Comparison of the diameter, cross-sectional area, and position of the left and right internal jugular vein and carotid artery in adults using ultrasound. J. Clin. Anesth, 32, 65-69 (2016).

30. Lulia, V., Petru, B. \& Viorel, I. Considerations on the origin of the ophthalmic artery from the internal carotid artery. ARS Med. Tomitana, 4, 179-183 (2018).

31. Chylack, L. T. Jr. et al. Lens opacities classification system II (LOCS II). Arch. Ophthalmol, 107, 991997 (1989).

32. Schulze-Bonsel, K., Feltgen, N., Burau, H., Hansen, L. \& Bach, M. Visual acuities "hand motion" and "counting fingers" can be quantified with the freiburg visual acuity test. Invest. Ophthalmol. Vis. Sci, 47, 1236-1240 (2006).

33. Hayreh, S. S., Klugman, M. R., Beri, M., Kimura, A. E. \& Podhajsky, P. Differentiation of ischemic from non-ischemic central retinal vein occlusion during the early acute phase. Graefes. Arch. Clin. Exp. Ophthalmol, 228, 201-217 (1990).

34. Brown, D. M. et al. RAVE Study Group. Ranibizumab in preproliferative (ischemic) central retinal vein occlusion: The rubeosis anti-VEGF (RAVE) trial. Retina, 34, 1728-1735 (2014).

35. Khayat, M., Williams, M. \& Lois, N. Ischemic retinal vein occlusion: Characterizing the more severe spectrum of retinal vein occlusion. Surv. Ophthalmol, 63, 816-850 (2018).

\section{Tables}

Table 1: Clinical characteristics of 517 eyes of 517 patients with CRVO at the initial visit to the hospital. 


\begin{tabular}{|lll|}
\hline Parameter & Value & $\begin{array}{c}P \text { - } \\
\text { value }\end{array}$ \\
\hline Number of eyes/subjects & $517 / 517$ & $69.9 \pm 12.2(22$ to 94$)$ \\
\hline Age, mean \pm SD (range), years & & $0.001^{\square}$ \\
\hline Sex & $296(57.3)$ \\
\hline Men (\%) & $221(42.7)$ \\
\hline Women (\%) & \\
\hline Affected eye & $240(46.4)$ \\
\hline Right (\%) & $277(53.6)$ \\
\hline Left (\%) & $334(64.6)$ \\
\hline Hypertension (\%) & $87(16.8)$ \\
\hline Diabetes Mellitus (\%) & $6.4 \pm 6.9(0$ to 51$)$ \\
\hline $\begin{array}{l}\text { Period from symptom onset to initial visit to hospital, mean } \pm \text { SD } \\
\text { (range), weeks }\end{array}$ & $0.72 \pm 0.55(-0.18$ to \\
\hline Best-corrected visual acuity, mean \pm SD (range), logMAR & $2.30)$ \\
\hline Central macular thickness, mean \pm SD (range), $\mu$ m & $632 \pm 237 \quad(62$ to \\
\hline Ischemic status at initial visit to hospital & $1456)$ \\
\hline Ischemic (\%) & $18(3.5)$ \\
\hline Nonischemic (\%) & \\
\hline Unclassifiable (\%) & $377(72.9)$ \\
\hline
\end{tabular}

SD, standard deviation; logMAR, logarithmic minimum angle of resolution. The probabilities of the appearance between men and women or between right and left were compared by a binomial test. ${ }^{\square}$ $P<0.05$.

Table 2: Factors affecting best-corrected visual acuity (in logMAR units) at the initial visit to the hospital in patients with CRVO. Results of univariable and multivariate linear regression analyses are shown. 


\begin{tabular}{lllll} 
& \multicolumn{2}{c}{$\begin{array}{l}\text { Univariate regression } \\
\text { analysis }\end{array}$} & \multicolumn{2}{c}{$\begin{array}{l}\text { Multivariate regression } \\
\text { analysis }\end{array}$} \\
\hline Independent variables & $r$ & $p$-Value & $\beta$ & $P$-Value \\
\hline Age (years) & 0.194 & $<0.001^{\star \star}$ & 0.191 & $<0.001^{\star}$ \\
\hline Sex (men/women) & 0.005 & 0.916 & 0.023 & 0.586 \\
\hline Affected eye (right/left) & -0.103 & $0.019^{\star}$ & -0.089 & $0.041^{\star}$ \\
\hline Hypertension & 0.035 & 0.432 & 0.013 & 0.764 \\
\hline Diabetes mellitus & -0.043 & 0.325 & -0.045 & 0.303 \\
\hline $\begin{array}{l}\text { Period from symptom onset to initial visit to } \\
\text { hospital (weeks) }\end{array}$ & & & & \\
& 0.008 & 0.848 & -0.008 & 0.849
\end{tabular}

Correlation coefficient $(r)$, standardized partial regression coefficient $(\beta)$, and $P$ value are shown for six independent variables, which can affect best-corrected visual acuity (logMAR unit) at the initial visit to the hospital in patients with CRVO. ${ }^{*} P<0.05$ was considered significant. ${ }^{*} P<0.01$.

\section{Figures}




\section{All patients}

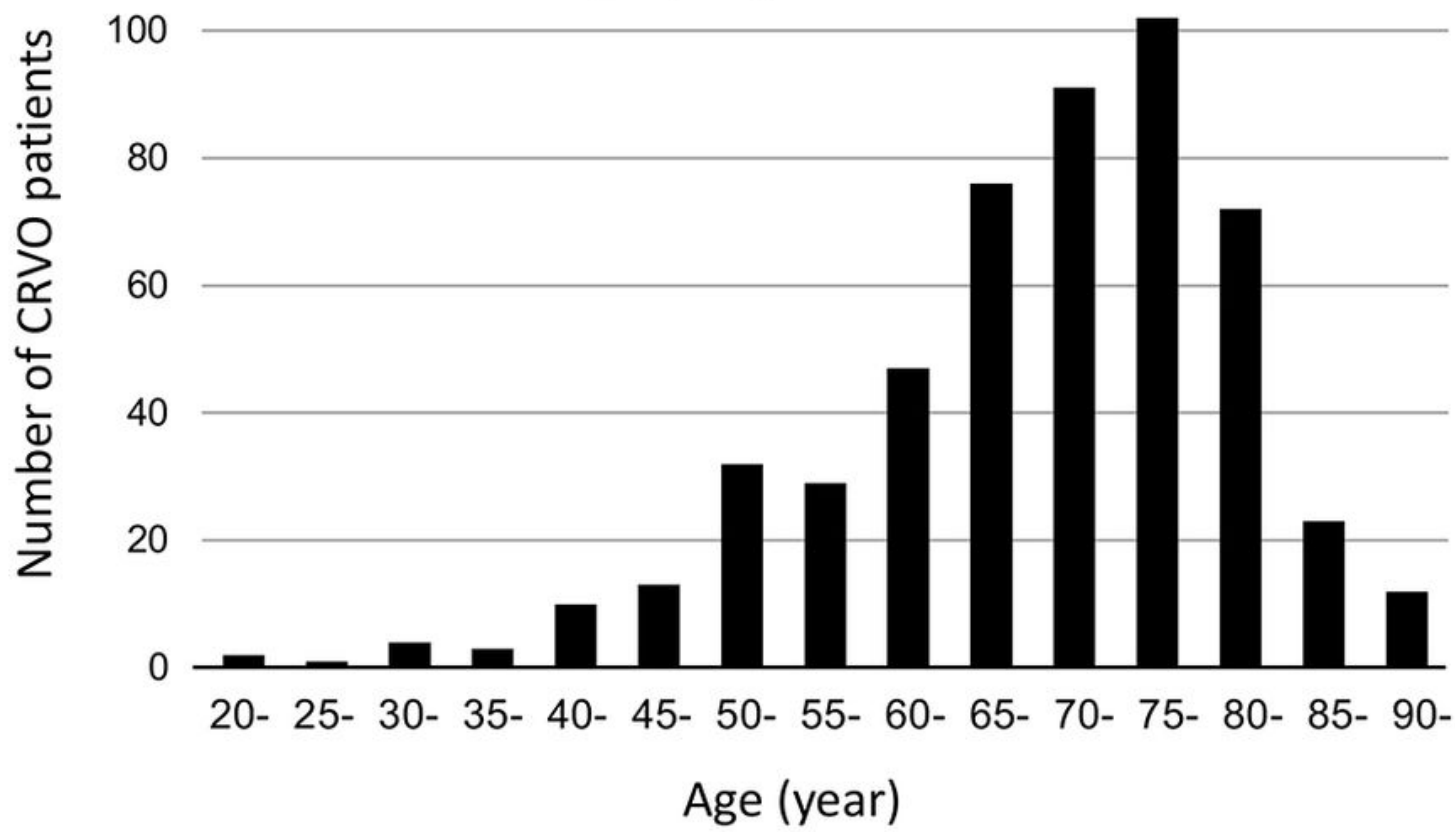

B

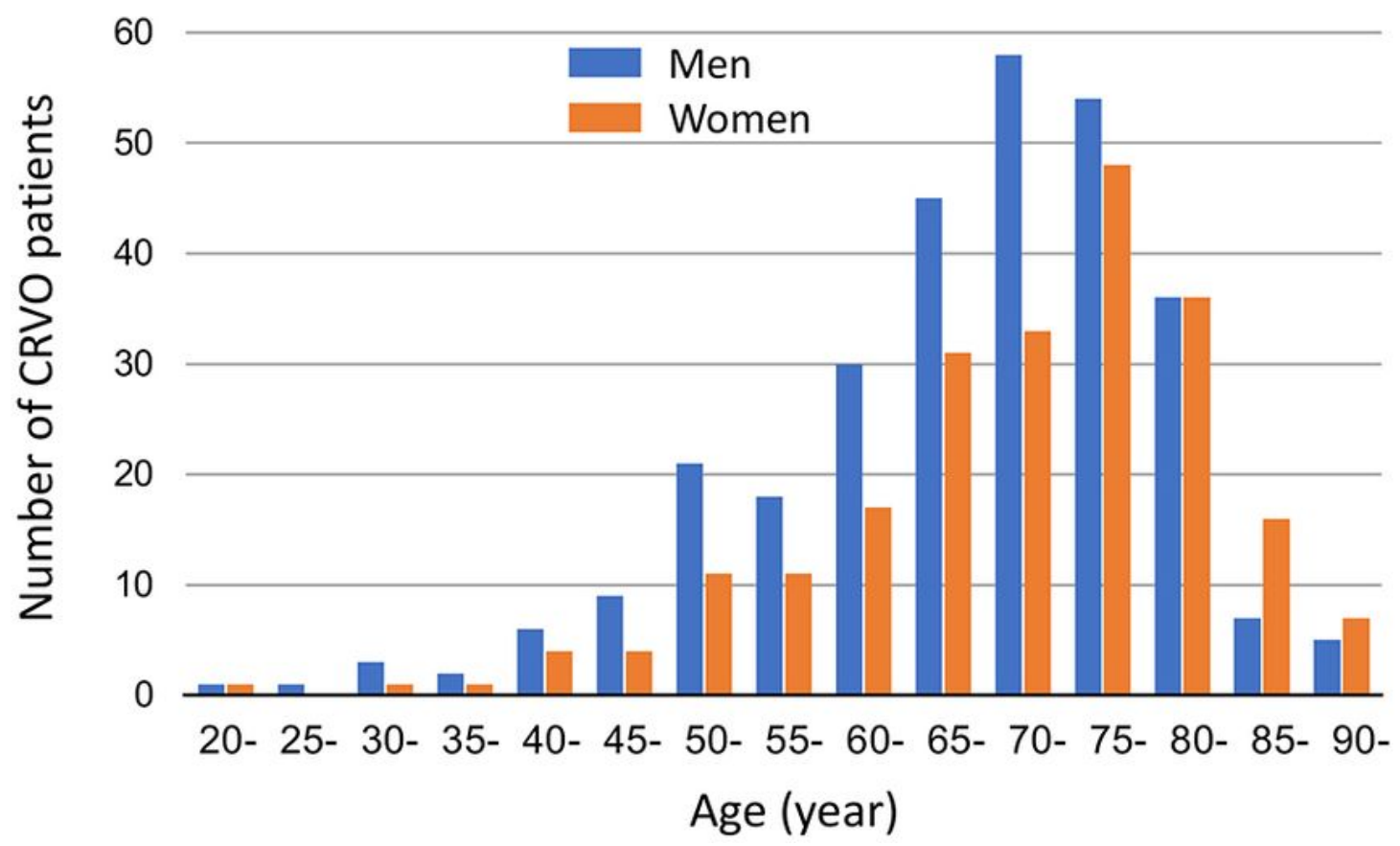

Figure 1

(A) Number of patients with central retinal vein occlusion (CRVO) for each age group. The mean \pm standard deviation (SD) age of the patients was $69.9 \pm 12.2$ years with a range of 22 to 94 years. There were $58.0 \%$ of the CRVO patients who were 70 years or older. (B) Number of patients with CRVO for each age group shown by sex. The mean age of the CRVO patients was significantly higher in women (71.9 \pm 12.1 years $)$ than in men $(68.4 \pm 12.1$ years, $P=0.001)$. 
A

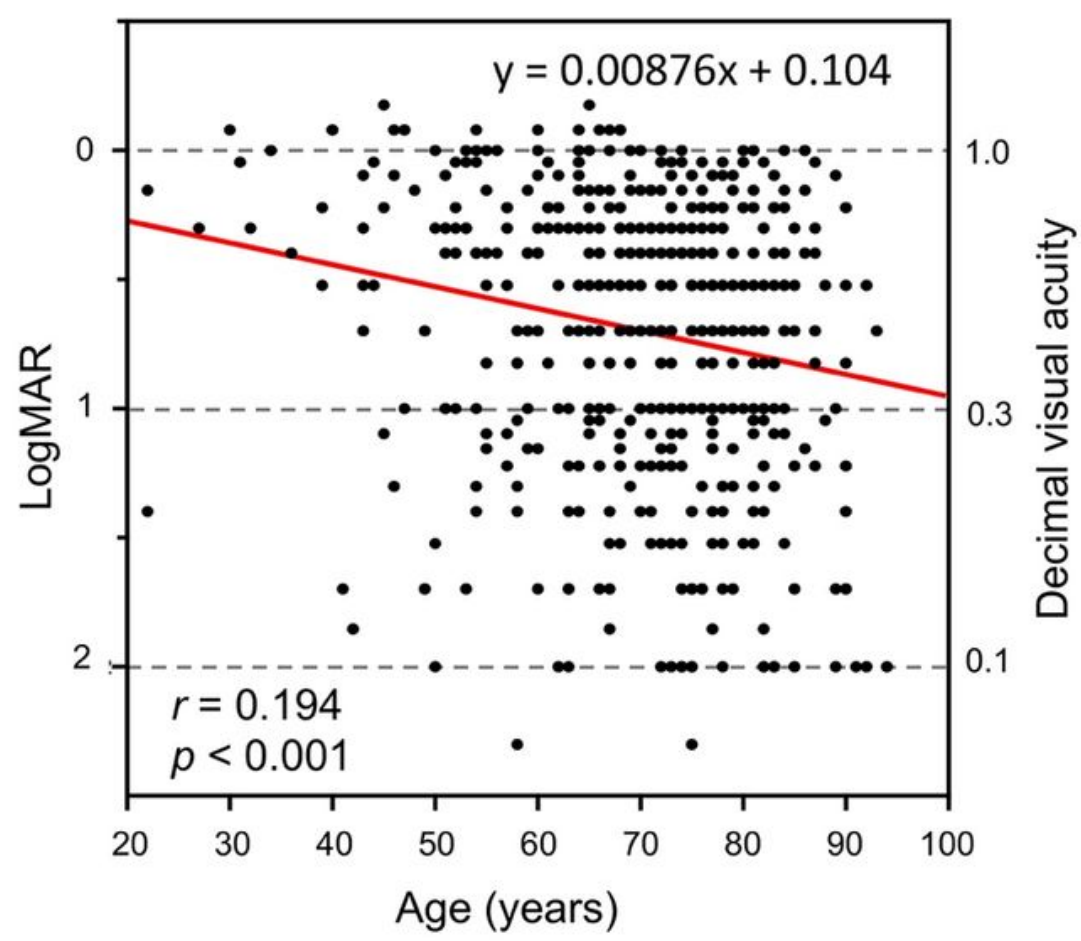

B

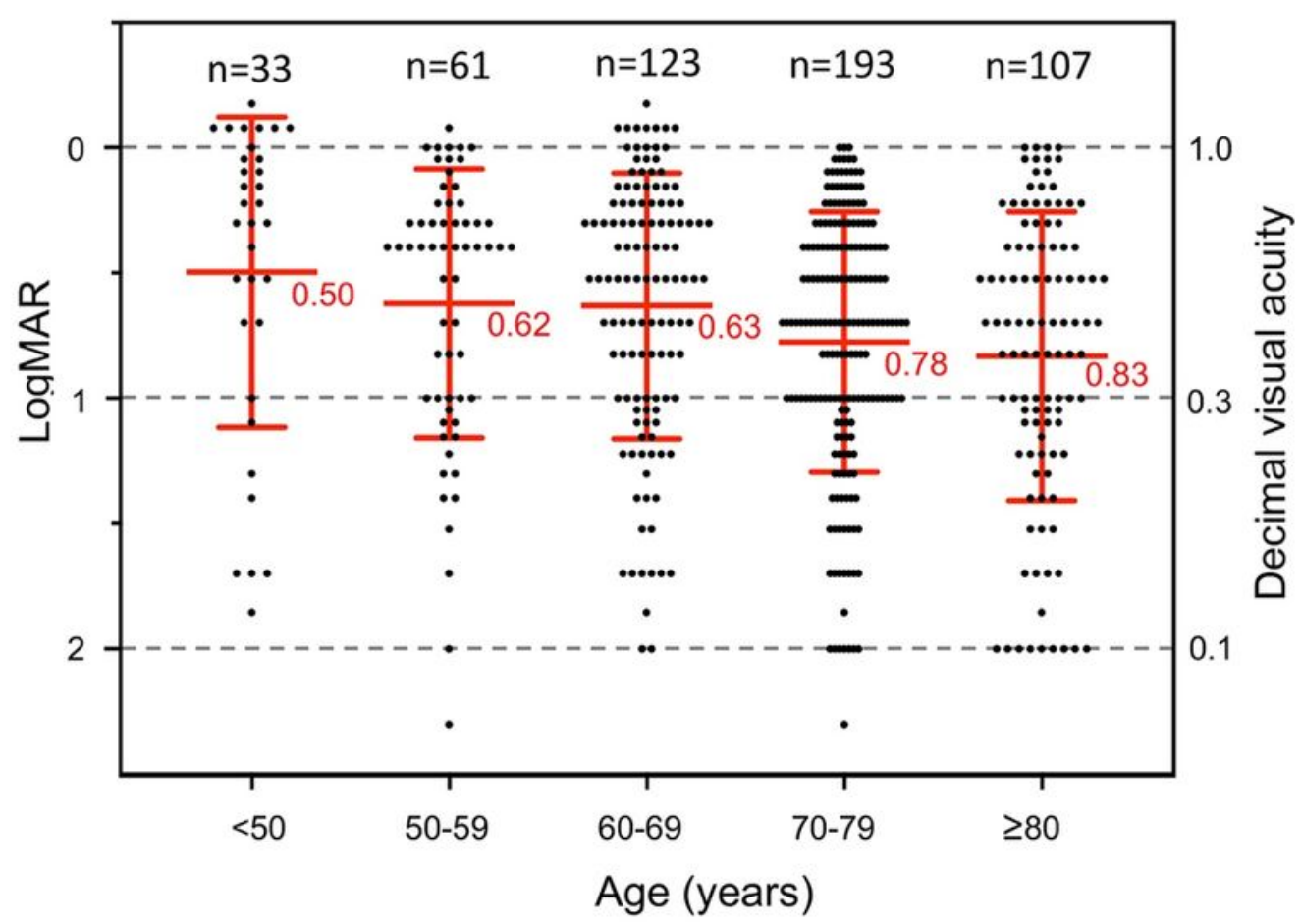

Figure 2

(A) The best-corrected visual acuity (BCVA) in logarithm of the minimal angle of resolution (logMAR) units at the initial visit to hospital plotted against the age. A linear regression fit to the data is shown by the red line $(r=0.194, P<0.001)$. (B) The BCVA (logMAR units) at the initial visit to the hospital plotted for each age group. The mean BCVA worsened with increasing age. The red bars indicate the means \pm SDs. 


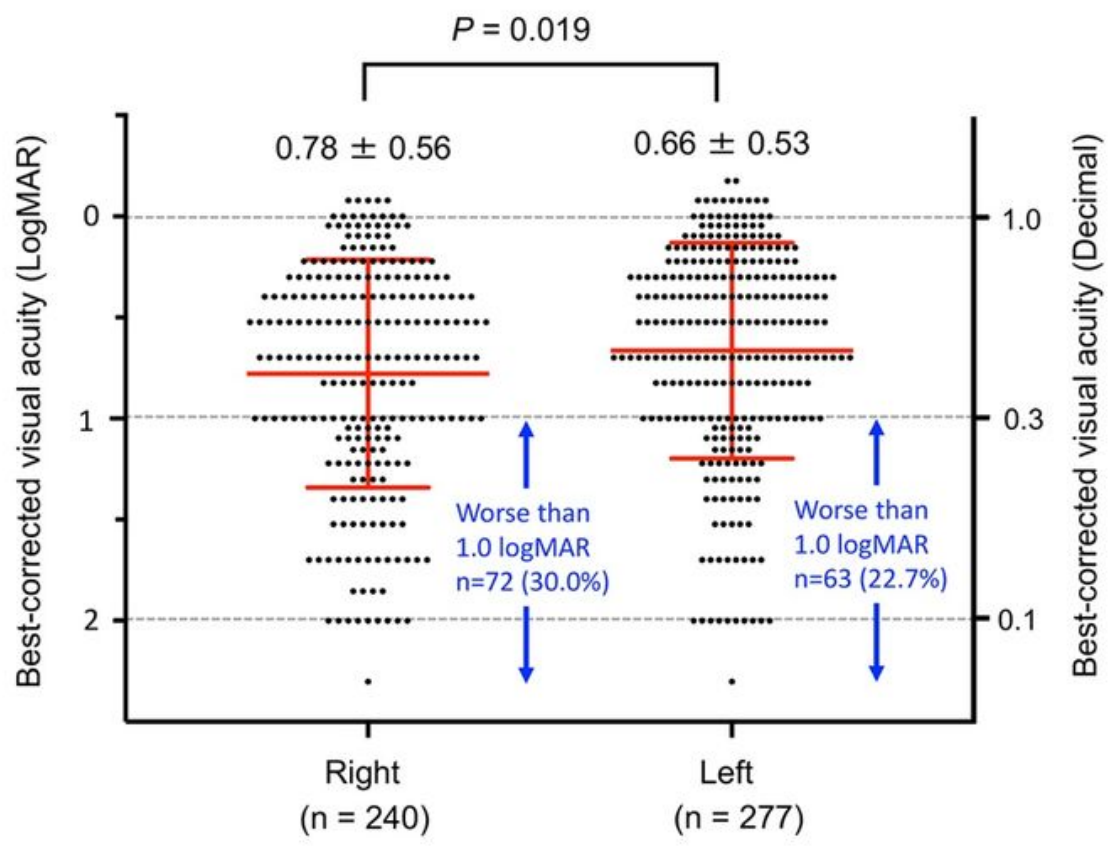

B

\section{Central Macular Thickness}

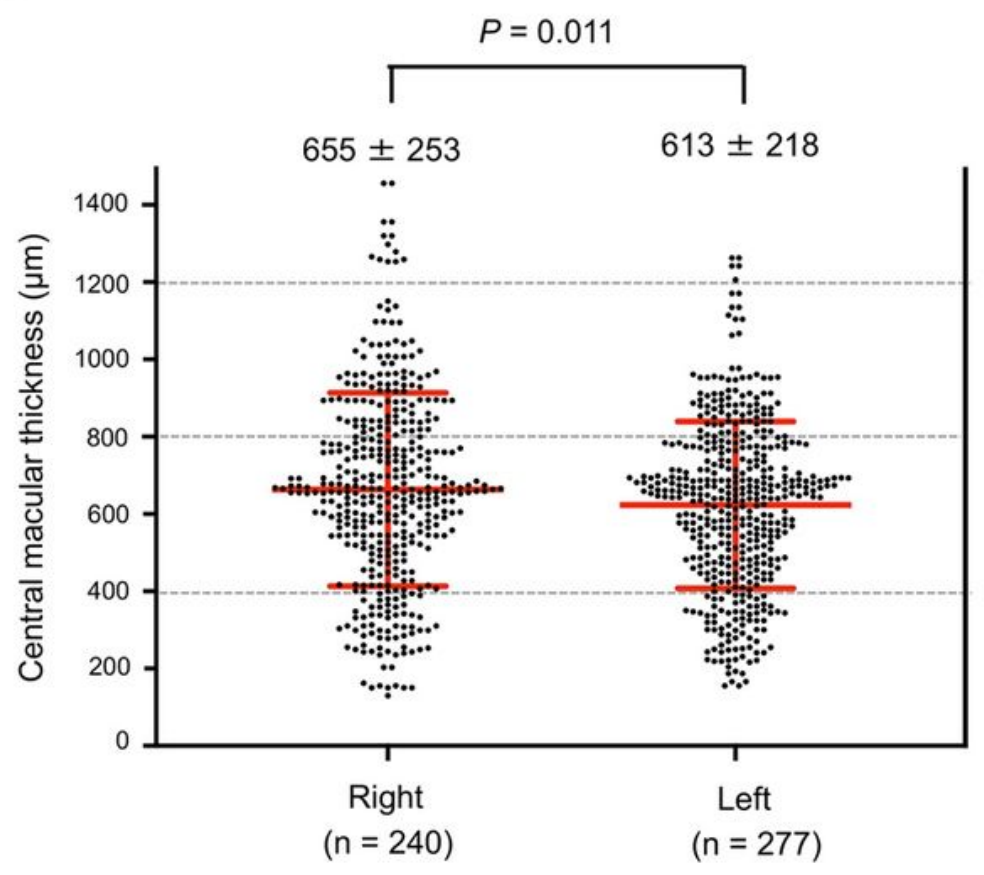

Figure 3

(A) Comparisons of the BCVA (in logMAR units) between the right and left eyes at the initial examination in all 517 CRVO patients. The mean \pm SD of the BCVA (logMAR unit) was significantly better in the left eye $(0.66 \pm 0.53)$ than in the right eye $(0.78 \pm 0.56, P=0.019)$. The percentage of eyes with BCVA worse than 1.0 logMAR units ( 0.1 decimal BCVA) was $30.0 \%$ (72/240 eyes) in the right eye, whereas it was $22.7 \%$ in the left eye (63/277 eyes). (B) Comparisons of central macular thickness (CMT) between the 
right and left eyes at the initial examination in all 517 CRVO patients. The mean CMT was significantly thicker in the right eye $(655 \pm 253 \mu \mathrm{m})$ than in the left eye $(613 \pm 218 \mu \mathrm{m}, \mathrm{P}=0.011)$.

\section{Supplementary Files}

This is a list of supplementary files associated with this preprint. Click to download.

- SI.docx 